\title{
Dysfunction of ATG7-dependent autophagy dysregulates the antioxidant response and contributes to oxidative stress-induced biological impairments in human epidermal melanocytes
}

\author{
Zhuhui Qiao', Zhongyi Xu $\mathbb{1}^{1}$, Qing Xiao ${ }^{1}$, Yiwen Yang ${ }^{1}$, Jiayi Ying ${ }^{1}$, Leihong Xiang ${ }^{1}$ and Chengfeng Zhang (1)
}

\begin{abstract}
Autophagy is a process involving the self-digestion of components that participates in anti-oxidative stress responses and protects cells against oxidative damage. However, the role of autophagy in the anti-oxidative stress responses of melanocytes remains unclear. To investigate the role of autophagy in human epidermal melanocytes, we knocked down and overexpressed ATG7, the critical gene of autophagy, in normal human epidermal melanocytes. We demonstrated that ATG7-dependent autophagy could affect melanin content of melanocytes by regulating melanogenesis. Moreover, suppression of ATG7-dependent autophagy inhibits proliferation and promotes oxidative stress-induced apoptosis of melanocytes, whereas enhancement of ATG7-dependent autophagy protects melanocytes from oxidative stress-induced apoptosis. Meanwhile, deficiency of ATG7-dependent autophagy results in premature senescence of melanocytes under oxidative stress. Notably, we verified that ATG7-dependent autophagy could alter oxidative stress homeostasis by regulating reactive oxygen species (ROS) production, nuclear factor erythroid 2-related factor 2 (Nrf2) antioxidant pathway, and the activity of several antioxidant enzymes in melanocytes. In conclusion, our study suggested that ATG7-dependent autophagy is indispensable for redox homeostasis and the biological functions of melanocytes, such as melanogenesis, proliferation, apoptosis, and senescence, especially under oxidative stress.
\end{abstract}

\section{Introduction}

Human skin is directly and constantly under the risks of numerous oxidative environmental stressors, such as ultraviolet (UV) irradiation, air pollutants, and chemical products. Redox balance between free radicals and antioxidants, which detoxify their harmful effects is critical for maintaining normal functions of various skin cell types, including keratinocytes, melanocytes, and fibroblast ${ }^{1-3}$. Excessive oxidative stress may lead to skin problems, especially aging ${ }^{4}$ and skin cancer ${ }^{5}$. Recently, oxidative

\footnotetext{
Correspondence: Leihong Xiang (flora_xiang@vip.163.com) or

Chengfeng Zhang (e3dangdang@hotmail.com)

'Department of Dermatology, Huashan Hospital, Fudan University, Shanghai,

China

These authors contributed equally: Zhuhui Qiao, Zhongyi Xu

Edited by: Inna Lavrik
}

stress has also come to light as a possible mechanism in autoimmune skin diseases ${ }^{6}$, inflammatory skin diseases ${ }^{7}$, and pigmentation skin disorders, such as vitiligo ${ }^{8-10}$. Theoretically, reactive oxygen species (ROS) can attack melanocytes and interfere with normal metabolism, proliferation, and differentiation of melanocytes, which ends up causing cell apoptosis and defects ${ }^{11}$. Studies have indicated that dysfunction of the anti-oxidative system in patients with vitiligo may increase the vulnerability of melanocytes to oxidative damage. Accumulation of ROS will further lead to the impairment of redox homeostasis and result in the destruction of melanocytes ${ }^{12,13}$. However, effective treatment options for vitiligo are still lacking $^{14}$, thus driving researchers to seek potential new therapies. 


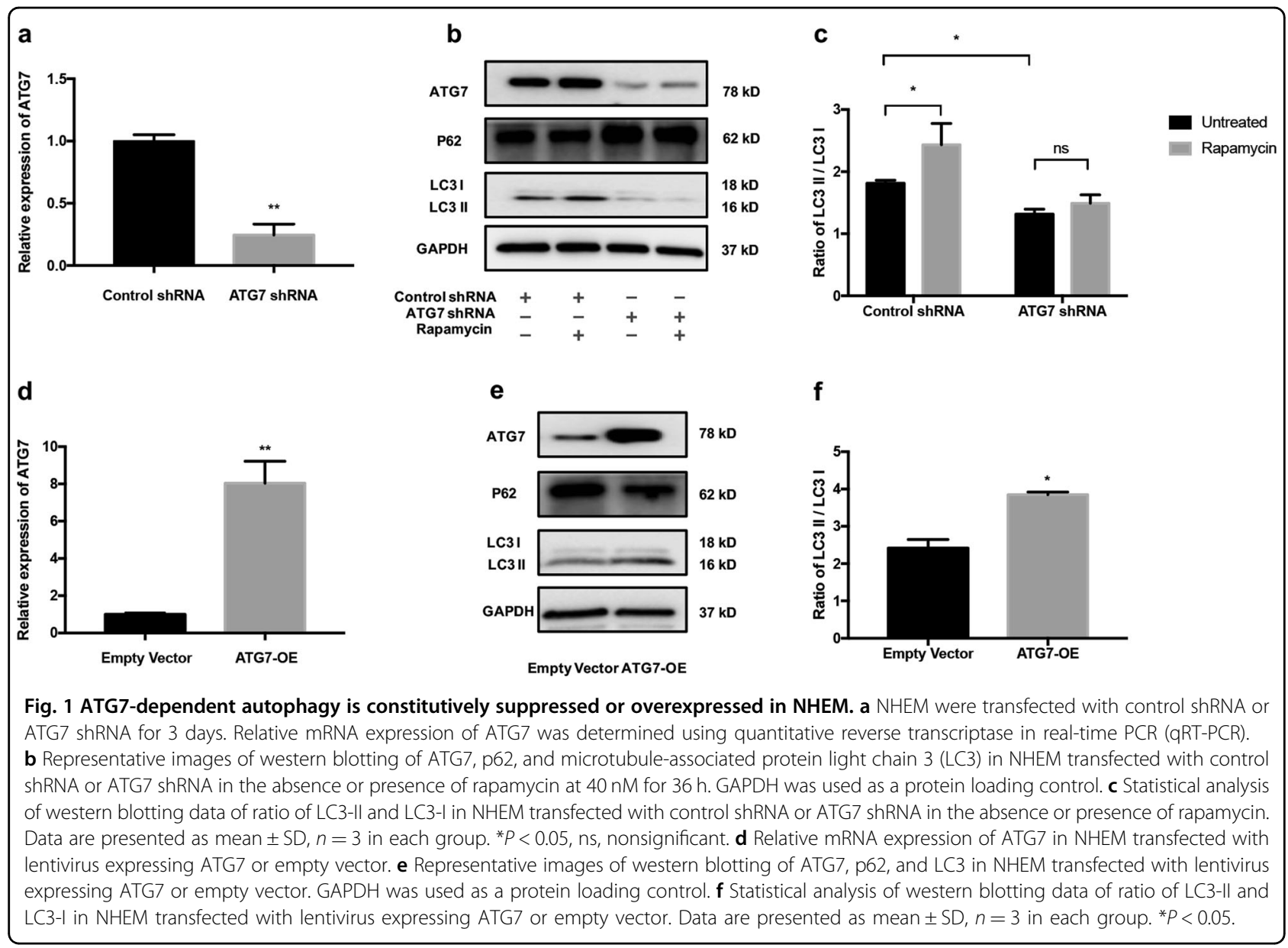

Autophagy, also known as "self-eating," is a lysosomaldependent degradation pathway that is widely present in eukaryotic cells. Under conditions of starvation, infection, or oxidative stress, it can regulate the degradation of longlived proteins and organelles in cells so that the degradation products can be reused by cells ${ }^{15}$. We previously revealed that autophagy plays a crucial role in the antioxidative stress response of skin keratinocytes ${ }^{1}$. Besides, we have confirmed that autophagy functions in both humans and murine melanocytes, and deficiency of ATG7-dependent autophagy can lead to diminished melanin content of dorsal hair, decreased proliferation and premature senescence of melanocytes in mice ${ }^{2}$. However, whether autophagy participates in redox homeostasis and the biological functions of natural human epidermal melanocytes (NHEMs) under oxidative stress remains unknown.

In this study, we aimed to investigate melanogenesis, proliferation, senescence, and oxidative stress response of autophagy-competent and autophagy-deficient NHEM. We showed that ATG7-dependent autophagy had a great influence on melanogenesis, proliferation, apoptosis, and senescence of NHEM, especially under oxidative stress.
Meanwhile, it altered oxidative stress homeostasis by regulating reactive ROS production, nuclear factor erythroid 2-related factor 2 (Nrf2) antioxidant pathway, and the activity of several antioxidant enzymes.

\section{Results}

\section{Knockdown or overexpression of ATG7 efficiently could} suppress or enhance autophagy in NHEM

To specifically knock down ATG7 gene in NHEM, the ATG7 short hairpin RNA (shRNA) and control shRNA were transfected. Quantitative reverse transcriptase in real-time PCR (qRT-PCR) analysis showed that the mRNA expression of ATG7 in NHEM was significantly decreased in ATG7 shRNA group (Fig.1a). Western blotting analysis further demonstrated that the protein expression of ATG7 in melanocytes was also diminished in ATG7 shRNA group. Meanwhile, the expression of the autophagy adapter protein p62 was increased and the conversion of LC3-I to LC3-II was decreased in ATG7 shRNA group compared with the control group. Upon rapamycin treatment, conversion ratio of LC3 increased in the control group, whereas the addition of rapamycin caused no increase in LC3-II in the 
ATG7 shRNA group (Fig. 1b, c). By contrast, ATG7 overexpression by lentivirus transfection significantly enhanced the mRNA and protein levels of ATG7, whereas the expression of p62 was downregulated in NHEM (Fig. 1d, e, f). Collectively, these data indicated that knockdown or overexpression of ATG7 could efficiently suppress or enhance autophagy in NHEM.

\section{Inactivation of ATG7-dependent autophagy affects melanin content and leads to dysfunction of melanogenesis}

To investigate whether autophagy was involved in melanin synthesis of NHEM, melanin content and expressions of some crucial genes for melanogenesis, such as tyrosinase (TYR), TYR-related protein 1 (TRP1), TYRrelated protein 2 (TRP2), and microphthalmia-associated transcription factor (MITF) were investigated. The relative melanin content of NHEM in ATG7 shRNA group (referred to autophagy-deficient later) was significantly lower than control group (Fig. 2a), whereas the relative melanin content of the ATG7-overexpression group (referred to autophagy-competent later) was significantly higher (Fig. 2a). In addition, the mRNA expressions of TYR, TRP1, TRP2, and MITF were significantly lower in ATG7 shRNA group than control group. The downregulation of TYR, TRP1, TRP2, and MITF was further confirmed by western blotting in NHEM transfected with ATG7 shRNA (Fig. 2b, c). On the contrary, when ATG7 was overexpressed in NHEM, both the mRNA and protein levels of TYR, TRP1, TRP2, and MITF were significantly increased in comparison with those in the empty vector group (Fig. 2d, e). Taken together, these results indicate that ATG7-dependent autophagy is crucial for melanogenesis and it is involved in the process of melanin biosynthesis.

\section{Deficiency of ATG7-dependent autophagy inhibits proliferation and facilitates oxidative stress-induced apoptosis of NHEM}

To explore the role of autophagy in oxidative stressinduced apoptosis, ATG7-transfected NHEM were stimulated with either hydrogen peroxide $\left(\mathrm{H}_{2} \mathrm{O}_{2}\right)$ or ultraviolet $B$ (UVB) irradiation. The growth curve of NHEM illustrated that suppression of ATG7-dependent autophagy inhibited the proliferation of NHEM not only under normal conditions but upon oxidative stress as well. However, overexpression of ATG7-dependent autophagy only promoted the proliferation of NHEM under oxidative stress (Fig. 3a). To further investigate how ATG7dependent autophagy inhibited cell proliferation, autophagy-deficient and autophagy-competent NHEM were treated with $\mathrm{H}_{2} \mathrm{O}_{2}$ or UVB irradiation. As shown in Fig. 3b, c, the average percentage of apoptotic cells was readily elevated $(43.13 \pm 2.53 \%$ vs. $27.73 \pm 1.52 \%)$ in autophagy-deficient NHEM in baseline level and was further elevated $(24.64 \pm 1.35 \%$ vs. $39.76 \pm 5.09 \%)$ after $\mathrm{H}_{2} \mathrm{O}_{2}$ treatment and $(67.83 \pm 5.76 \%$ vs. $45.27 \pm 2.12 \%)$ upon UVB irradiation. Moreover, ATG7 knockdown could upregulate the level of cleaved Poly(ADP-ribose) polymerase1(PARP), a nuclear enzyme involved in DNA repair, and cleaved caspase 3 (Fig. 3d, e). By contrast, when autophagy-competent NHEM were treated with $\mathrm{H}_{2} \mathrm{O}_{2}$ or UVB irradiation, the average percentage of apoptotic cells was significantly reduced ( $24.77 \pm 1.00 \%$ vs. $43.97 \pm 3.49 \%$ and $25.37 \pm 1.10 \%$ vs. $44.10 \pm 1.65 \%)$ (Fig. 3f, g), in combination with decreased level of cleaved PARP and cleaved caspase 3 (Fig. 3h, i). These results indicate that deficiency of ATG7-dependent autophagy could facilitate oxidative stress-induced apoptosis.

\section{Deficiency of ATG7-dependent autophagy results in oxidative stress-induced premature senescesnce of NHEM}

Next, we characterized the impact of ATG7-dependent autophagy on the aging of NHEM. Autophagy-deficient NHEM started to change morphology early in the third passage and acquired senescent morphotypes with distended cytoplasms. The premature senescent phenotype became even more prominent with the treatment of $\mathrm{H}_{2} \mathrm{O}_{2}$ and UVB irradiation compared with control group. By contrast, no morphology change was observed in autophagy-competent NHEM in baseline condition or under oxidative stress (Fig. 4a). Subsequently, we analyzed two important senescence markers in the transfected NHEM. The activity of senescence-associated $\beta$-galactosidase (SA $\beta$-Gal) was dramatically increased in autophagy-deficient NHEM in baseline condition or under oxidative stress, whereas the SA $\beta$-Gal activity was dramatically decreased in autophagy-competent NHEM only under the treatment of $\mathrm{H}_{2} \mathrm{O}_{2}$ or UVB irradiation (Fig. 4b, c). Moreover, knockdown of ATG7 significantly increased the mRNA level of p16, whereas overexpression of ATG7 could decrease the mRNA expression of p16 in NHEM under the treatment of $\mathrm{H}_{2} \mathrm{O}_{2}$ or UVB irradiation (Fig. 4d). These results suggest that impairment of ATG7dependent autophagy leads to premature senescence of NHEM. Autophagy may protect NHEM from oxidative stress-induced premature senescence.

\section{ATG7-dependent autophagy protects NHEM from ROS generation, while deficiency of ATG7-dependent autophagy is responsible for activation of the Nrf2-ARE signaling pathway in NHEM under oxidative stress}

We next identified the regulatory role of ATG7dependent autophagy in ROS production in NHEM. First, autophagy-deficient NHEM were treated with $\mathrm{H}_{2} \mathrm{O}_{2}$ or UVB irradiation. The results showed that knockdown of ATG7-dependent autophagy could accelerate the generation of excessive ROS induced by $\mathrm{H}_{2} \mathrm{O}_{2}$ and UVB 

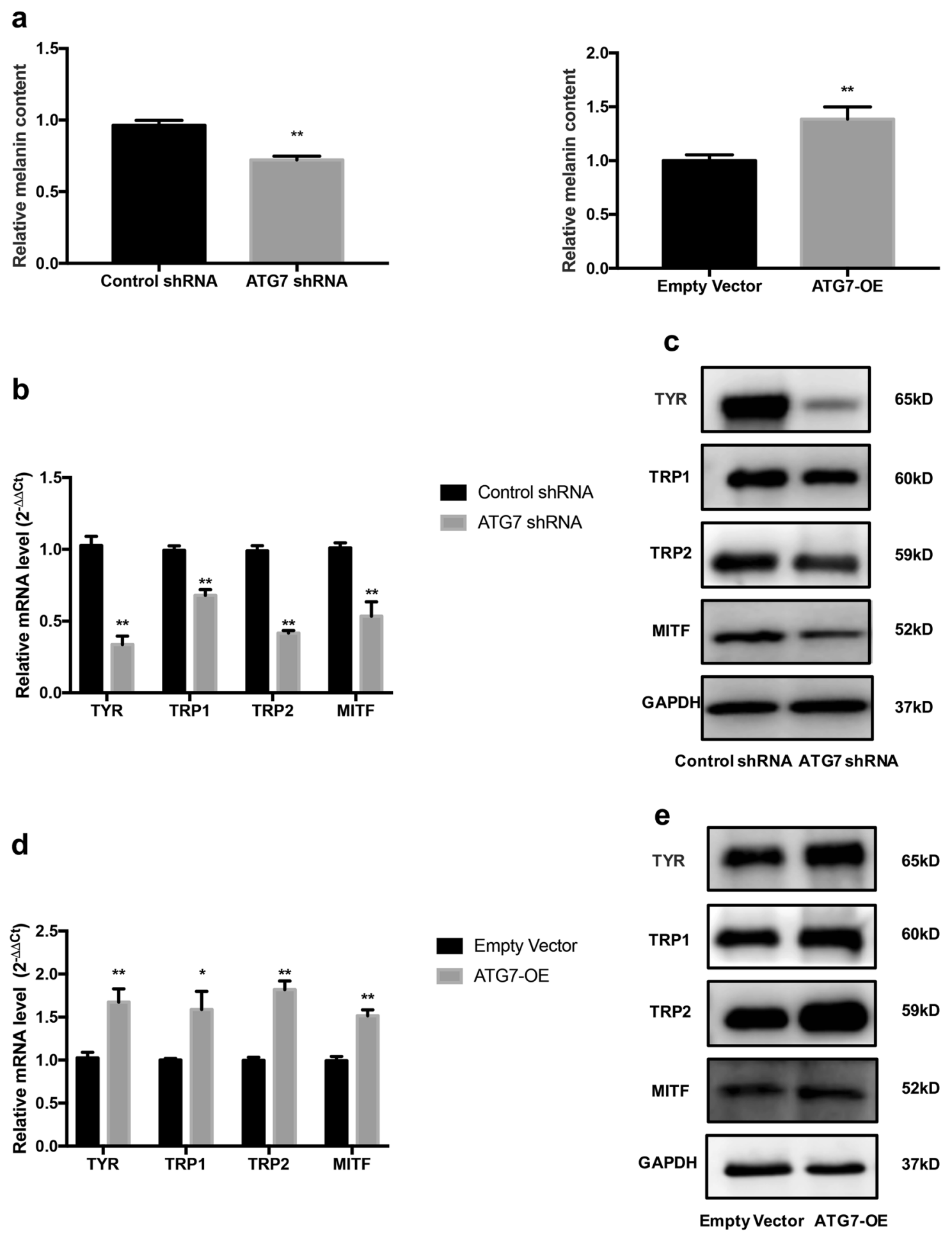

Fig. 2 ATG7-dependent autophagy regulates melanin content and melanogenesis of NHEM. a Melanin contents of NHEM transfected with control shRNA, ATG7 shRNA, empty vector, or lentivirus ATG7. b Relative mRNA expression of TYR, TRP1, TRP2, and MITF in NHEM transfected with control shRNA or ATG7 shRNA were detected by GRT-PCR. c Representative images of western blotting of TYR, TRP1, TRP2, and MITF in NHEM transfected with control shRNA or ATG7 shRNA. GAPDH was used as a protein loading control. d Relative mRNA expression of TYR, TRP1, TRP2, and MITF in NHEM transfected with empty vector or lentivirus ATG7 were detected by qRT-PCR. e Representative images of western blotting of TYR, TRP1, TRP2, and MITF in NHEM transfected with empty vector or lentivirus ATG7. GAPDH was used as a protein loading control. The data represent the mean \pm SD from three independent experiments. ${ }^{*} P<0.05$, ${ }^{* *} P<0.01 ; n s$, nonsignificant. 


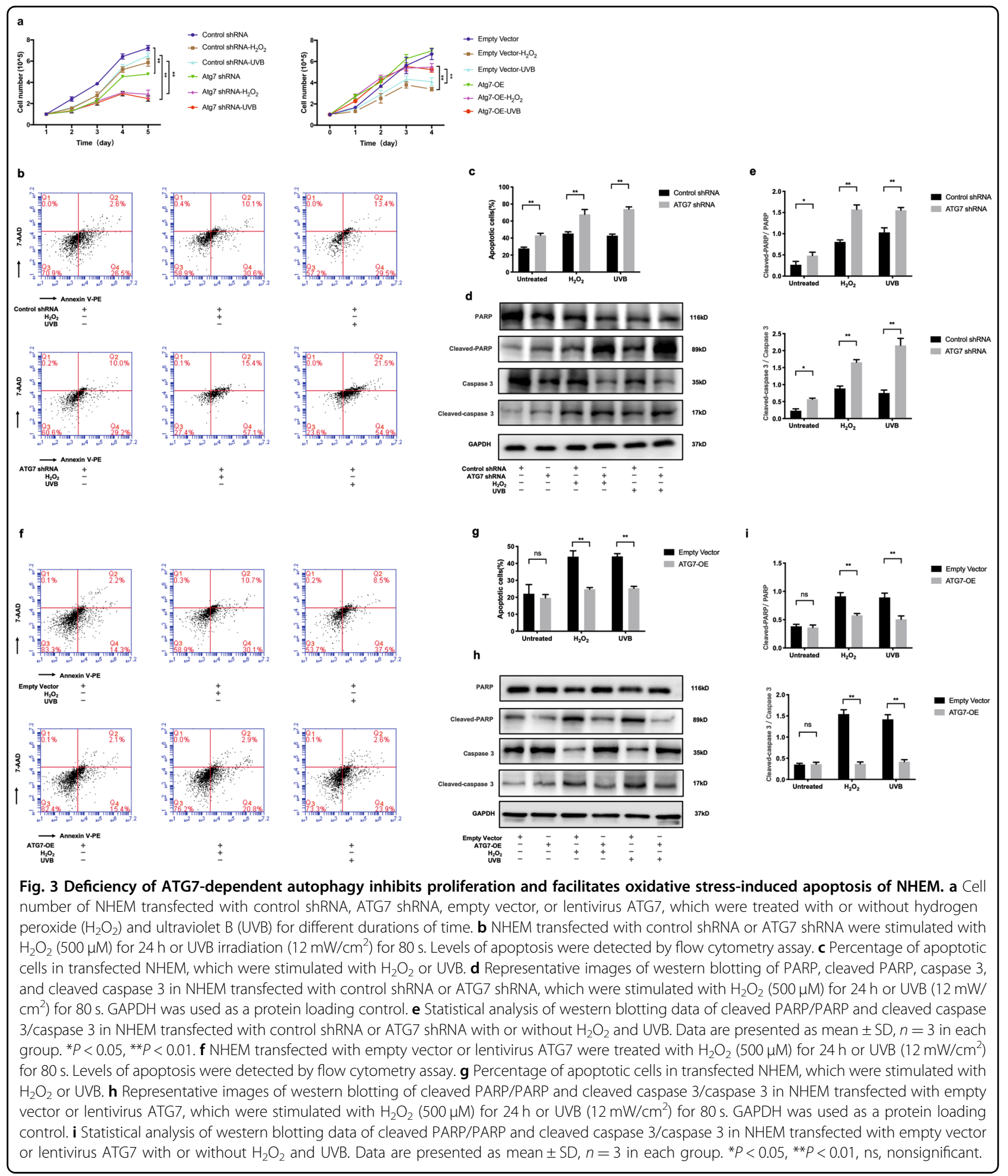

treatment. Meanwhile, we performed the same treatment on autophagy-competent NHEM. The results showed that the ROS induced by $\mathrm{H}_{2} \mathrm{O}_{2}$ and UVB irradiation was significantly decreased in the autophagy-competent NHEM compared with the control (Fig. 5a). Taken together, the above data revealed that ATG7-dependent autophagy plays a critical role in protecting NHEM from oxidative stress-induced ROS production.

In autophagy-deficient cells, an accumulation of the adapter protein $\mathrm{p} 62$ can be observed frequently. 


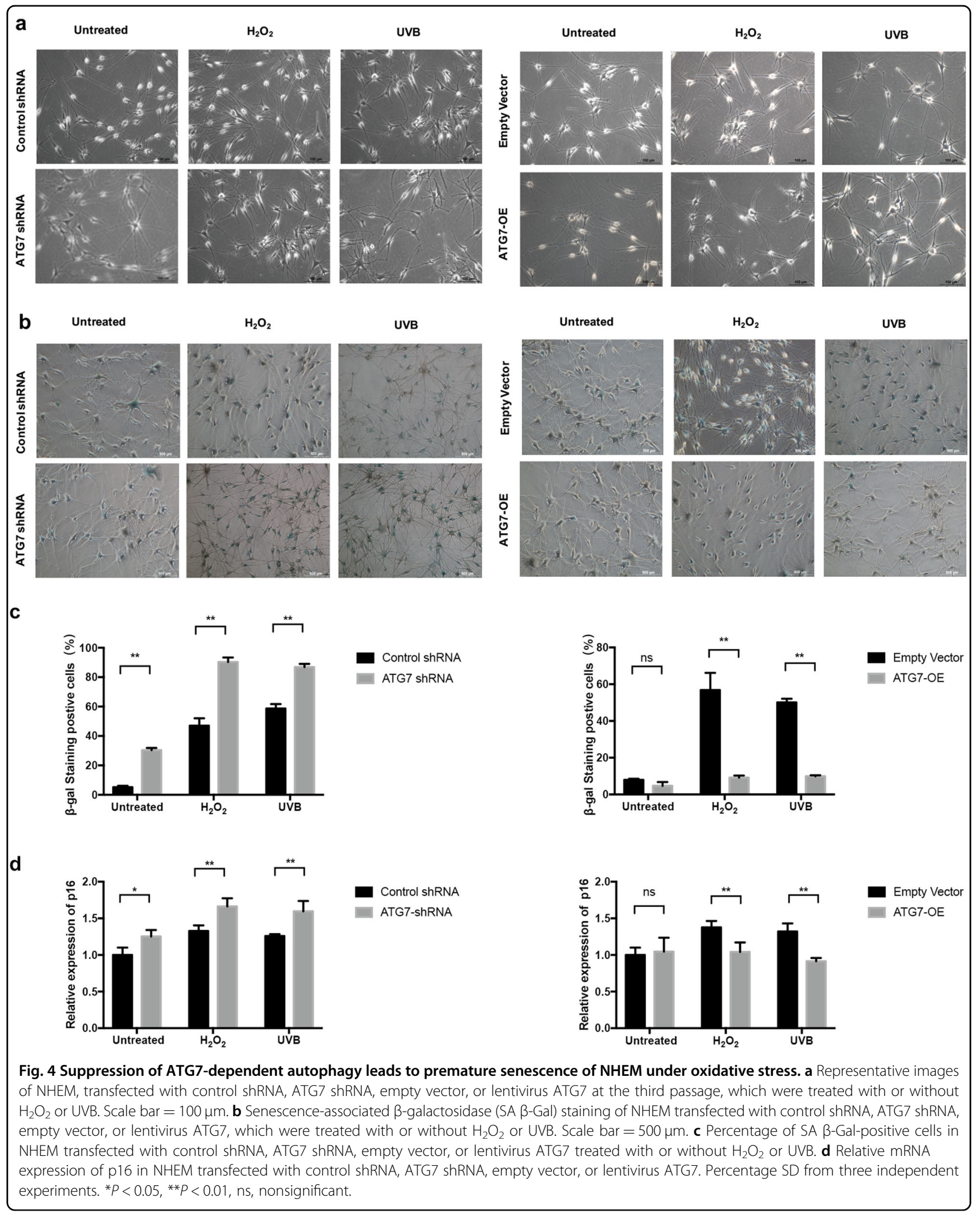




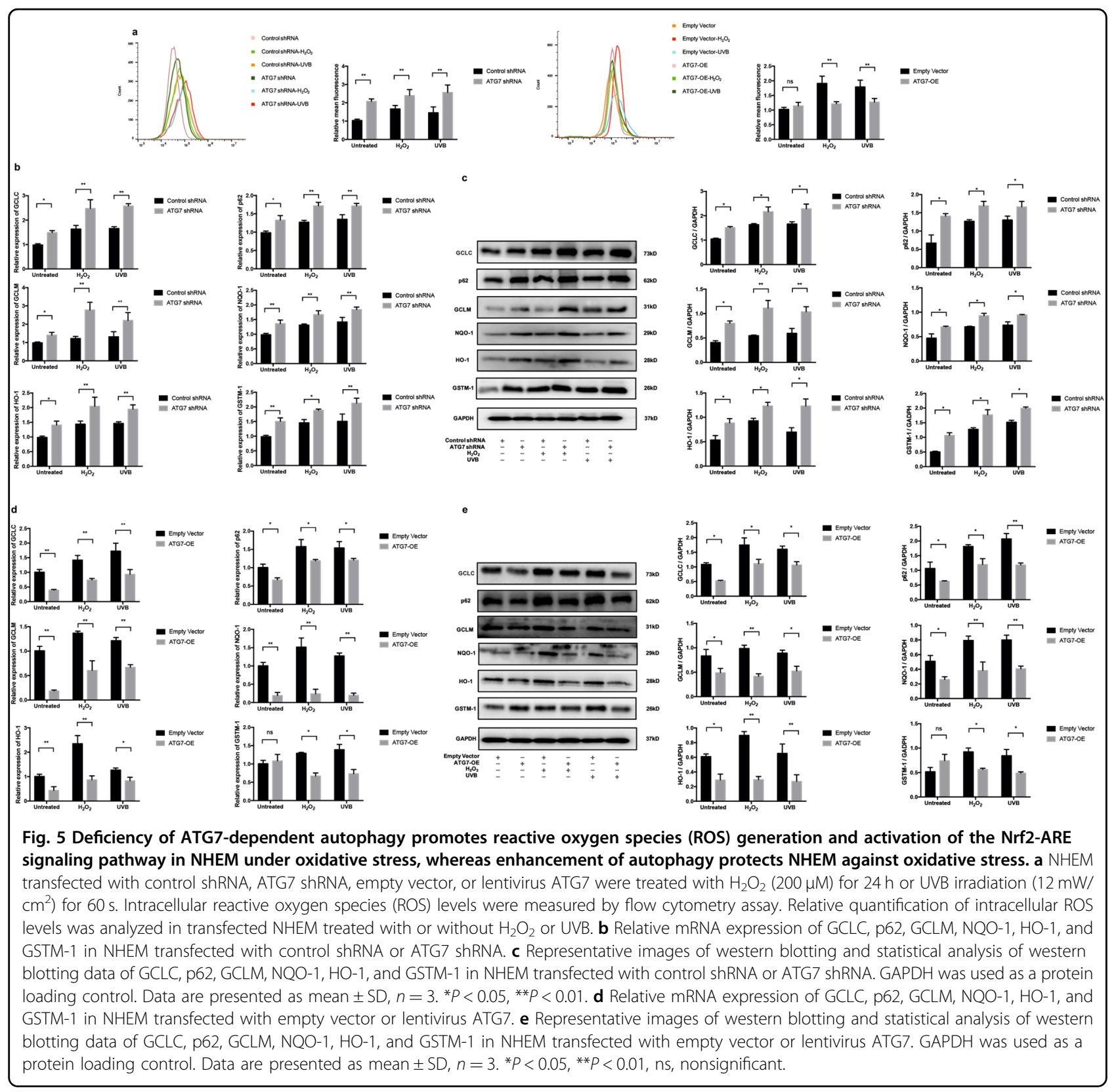

However, p62 is not only the adapter between cargo and the autophagic machinery but also a component of the Nrf2 (nuclear factor (erythroid-derived 2)-like 2)/ARE (antioxidant responsive element) signaling pathway, which is the antioxidant response that provides cellular antioxidants and detoxifying enzymes. Subsequently, we investigated whether the Nrf2 target genes were altered in autophagy-deficient NHEM. Indeed, qRT-PCR analysis showed that the canonical Nrf2 target genes, including $\gamma$-glutamyl cysteine ligase (GCLC), SQSTM1/ p62, glutamyl cysteine ligase modulatory subunit (GCLM), NAD(P)H dehydrogenase, quinone 1 (NQO-1), heme oxygenase-1 (HO-1), and glutathione $S$-transferase
Mu 1 (GSTM-1) were expressed at significantly higher levels in autophagy-deficient NHEM, both under normal conditions and oxidative stress (Fig. 5b). Western blotting analysis further confirmed that the protein levels of GCLC, p62, GCLM, NQO-1, HO-1, and GSTM-1 were increased both under normal conditions and oxidative stress (Fig. 5c). By contrast, the mRNA and protein levels of GCLC, p62, GCLM, NQO-1, HO-1, and GSTM-1 were significantly lower in autophagy-competent NHEM, both under normal condition and oxidative stress (Fig. 5d, e). These data indicate that the dysfunction of ATG7-dependent autophagy results in increased ROS production in NHEM. Autophagy is responsible for 


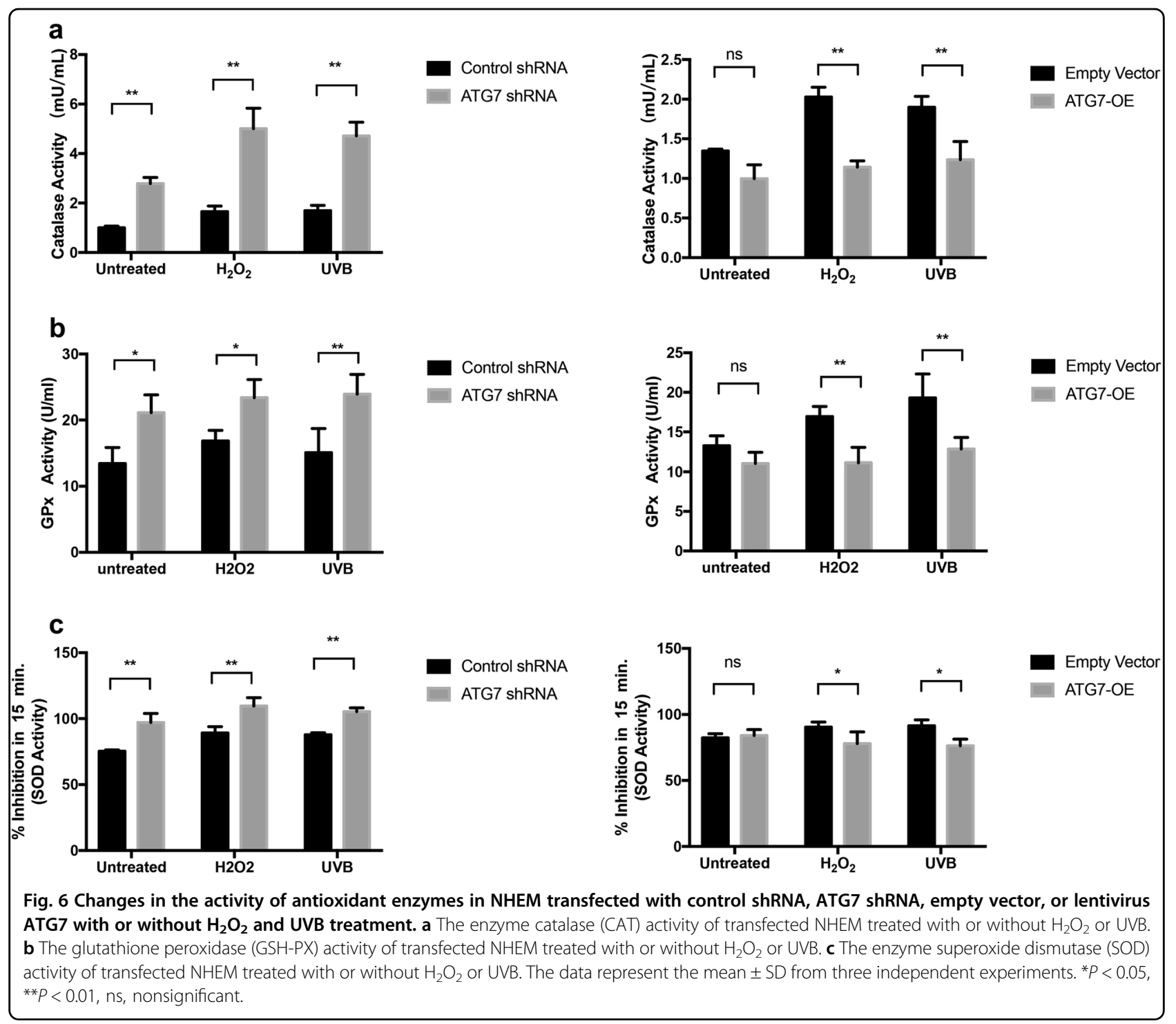

the regulation of the Nrf2-ARE signaling pathway under oxidative stress in NHEM.

\section{ATG7-dependent autophagy causes depletion of} antioxidant defense components under oxidative stress

Finally, we tried to investigate whether ATG7dependent autophagy could reduce cytotoxicity by promoting antioxidant defense components under oxidative stress. We revealed that the catalase (CAT) activity was increased in autophagy-deficient NHEM, both under normal condition and oxidative stress (Fig. 6a). However, the CAT activity had no difference between the ATG7overexpression group and the empty vector group under normal conditions. Only under oxidative stress such as $\mathrm{H}_{2} \mathrm{O}_{2}$ stimulation or UVB irradiation, the CAT activity in autophagy-competent NHEM was significantly decreased compared with control group. In addition, the glutathione peroxidase (GPx) and superoxide dismutase (SOD) activity were significantly increased in autophagydeficient NHEM, both under normal condition and oxidative stress (Fig. 6b, c). However, the GPx and SOD activity had no significant difference between autophagycompetent NHEM and control group under normal condition. Only under oxidative stress such as $\mathrm{H}_{2} \mathrm{O}_{2}$ stimulation or UVB irradiation, the GPx and SOD activity in autophagy-competent NHEM were significantly decreased (Fig. 6b, c). All the above data demonstrate that ATG7-dependent autophagy is involved in the balance between oxidative stress and antioxidant defense system.

\section{Discussion}

In this study, we investigated the role of ATG7dependent autophagy in the biological function and 
oxidative stress responses of primary human melanocytes under oxidative stress.

As is known to all, the primary role of melanocytes is melanin biosynthesis. The destruction of melanocytes is a crucial event in depigmentation skin disorders, such as vitiligo $^{16,17}$. Autophagy has been hypothesized to participate in melanogenesis ${ }^{18,19}$. Here we knocked down ATG7, the central regulator of autophagy, in NHEM and investigated its role in melanogenesis. Our results demonstrated that the genetic knockdown of ATG7-dependent autophagy in NHEM resulted in the reduction of melanin content and dysfunction of melanogenesis due to its suppression on TYR, TRP1, TRP2, and MITF, whereas the genetic overexpression of ATG7-dependent autophagy achieved the opposite results. In that case, alteration of ATG7-dependent autophagy might be involved in the pathogenesis of pigmentation diseases owing to its impact on melanogenesis.

In addition to melanogenesis, the decreased proliferative capacity and apoptosis of melanocytes are crucial in oxidative stress-induced depigmentation. Former studies have confirmed that apoptosis of melanocytes is common in patients with unstable vitiligo, which could be induced by melanocyte detachment and low adhesion ${ }^{20}$. Our study has demonstrated that autophagy-deficient melanocytes differed considerably from normal melanocytes in their proliferative capacity. Suppression of ATG7dependent autophagy significantly reduces the cell growth rate of melanocytes. In addition, impairment of autophagy renders melanocytes to oxidative stress-induced apoptosis. On the contrary, overexpression of ATG7-dependent autophagy efficiently maintains the proliferative capacity of melanocytes and reduces oxidative stress-induced apoptosis. These findings are in line with earlier reports on lung cancer cells that autophagy suppression may result in inhibition of proliferation and sensitize them to cell apoptosis ${ }^{21}$.

Skin cells are directly exposed to active oxygen species in the environment and are vulnerable to oxidative stressinduced senescence, which results in abnormal aging. Previous studies have revealed that oxidative stress can increase the production of ROS, which in turn aggravates the oxidative damage to the mitochondria ${ }^{22}$. Our study aims to investigate whether autophagy is responsible for protecting NHEM from oxidative stress-induced senescence. Our results indicated that autophagy-deficient melanocytes acquired senescent phenotype much earlier than normal control and lost the ability to fight against premature senescence under oxidative stress, which was consistent with earlier studies ${ }^{23}$. However, the specific molecular mechanisms regulating autophagy-dependent premature senescence under oxidative stress were still unclear. One study has reported the critical role of the SIRT3/AMPK signaling pathway in autophagy activation in the presence of oxidative stress in macrophages ${ }^{24}$, but further studies in melanocytes are still required.

Keap1/Nrf2/ARE signaling pathway is proved to be a critical antioxidant response in protecting melanocytes from oxidative damage ${ }^{25}$. Previous investigations on vitiligo patients have demonstrated that transcript levels of Nrf2 and the downstream detoxification genes such as GCLC, p62, GCLM, NQO-1, HO-1, and GSTM-1 were upregulated to protect melanocytes against $\mathrm{H}_{2} \mathrm{O}_{2}$-induced toxicity in vitiligo lesions compared with the matched non-lesional skin ${ }^{26,27}$. Recently, several evidence also support the connection between autophagy and Nrf2/ Keap1 signaling pathway by protein $\mathrm{p} 62^{28}$, but the underlying mechanisms are still not fully understood. During autophagy, intracellular proteins and organelles can be banded to autophagosomes by the adaptor protein p62; meanwhile, the $p 62 / S Q S T M 1$ is a target gene of Nrf2/Keap1 and creates a positive feedback loop between autophagy and Nrf2/Keap $1^{29,30}$. Komatsu et al. ${ }^{31}$ also found that the accumulation of p62 in autophagyknockout mice lead to overactivation of the Nrf2 pathway in liver cells and maintaining the stability of autophagy state plays an important role in protecting liver cells from damage stimulated by oxidative stress. On the other hand, the impairment of the Nrf2/Keap1 pathway can lead to defects of autophagy in vitiligo melanocytes ${ }^{32}$. Thus, we can summarize that the impairment of the Nrf2/Keap1 pathway induces the suppression of autophagy while autophagy deficiency leads to the overexpression of the Nrf2/Keap1 pathway. In accordance with the above statements, our study demonstrated that suppression of autophagy enhances activation of the Nrf2/ Keap1 signaling and attenuates the ability of NHEM to remove oxidative stress-induced ROS production. These findings strengthen the importance of ATG7-dependent autophagy in melanocytes homeostasis under oxidative stress, providing a potential target for treating depigmentation diseases such as vitiligo.

SOD, GPx, and CAT are important antioxidation enzymes for limiting ROS release and maintaining the integrity of cellular membrane construction ${ }^{33}$. In this study, we assayed the changes in SOD, GPx, and CAT activity. Autophagy deficiency could generate toxicity to cells and promote the activity of CAT, SOD, and GPx so as to decrease ROS accumulation. However, ROS might not be scavenged by elevated CAT, SOD, and GPx instantly and effectively, yet ended up with a significant elevation. Meanwhile, the levels of SOD, CAT, and GPx activity are inconsistent with previous in vivo studies on vitiligo $^{34-36}$, which indicates that the activity of antioxidation enzymes is changing dynamically at different stages of vitiligo. Thus, these results implied that ATG7dependent autophagy could influence the activity of antioxidation enzymes, but specific mechanisms of how 
Table 1 Sequences of primers for the quantitative RT-PCR (human).

\begin{tabular}{|c|c|c|c|}
\hline & Name & Forward & Reverse \\
\hline Human & p62 & 5'-AGT CGG ATA ACT GTT CAG GAG-3' & 5'-ATT CTG GCA TCT GTA GGG A-3' \\
\hline Human & GCLC & 5'-GTG GAT GTG GAC ACC AGA T-3' & 5'-GTC TTG CTT GTA GTC AGG AT-3' \\
\hline Human & GCLM & 5'-TGT ATC AGT GGG CAC AGG TA-3' & 5'-CAG TCA AAT CTG GTG GCA TC-3' \\
\hline Human & NQO-1 & $5^{\prime}$-GAG ACA GCC TCT TAC TTG CC-3' & 5'-AAA CCA CCA GTG CCA GTC A-3' \\
\hline Human & Ho-1 & 5'-ATT CTC TTG GCT GGC TTC CT-3' & 5'-CCT GGA TGT GCT TाT CGT TG-3' \\
\hline Human & GSTM-1 & 5'-GGG GAC GCT CCT GAT TAT GA-3' & $5^{\prime}$-CGG GCA ATG TAG CAC AAG A-3' \\
\hline Human & $\beta$-Actin & 5'-AAG GTG ACA GCA GTC GGT T-3' & 5'-TGT GTG GAC TTG GGA GAG G-3' \\
\hline Human & ATG7 & 5'-CTG CCA GCT CGC TTA ACA TTG-3' & $5^{\prime}-\mathrm{CTT}$ GTT GAG GAG TAC AGG GTT Tा-3' \\
\hline Human & GAPDH & 5'-AGA AGG CTG GGG CTC ATT TG-3' & 5'-AGG GGC CAT CCA CAG TCT TC-3' \\
\hline Human & p16 & 5'-GAT TGA AAG AAC CAG AGA GGC-3' & 5'-GAC CTT CGG TGA CTG ATG AT-3' \\
\hline Human & TRP1 & $5^{\prime}-\mathrm{TGG}$ CAA AGC GCA CAA CTC ACC C-3' & 5'-AGT GCA ACC AGT AAC AAA GCG CC-3' \\
\hline Human & TRP2 & 5'-TGG GAA ACT GTC TGT GAT AGC-3' & 5'-CCA TIT GAT TTC TC TCA GCA-3' \\
\hline Human & MITF & 5'-ATG CTG GAA ATG CTA GAA TAT AAT-3' & 5'-ATC ATC CAT CTG CAT ACA G-3' \\
\hline Human & TYR & 5'-TGG CAT AGA CTC TTC TTG TTG CGG-3' & 5'-CAA GGA GCC ATG ACC AGA TCC G-3' \\
\hline
\end{tabular}

autophagy regulates antioxidation enzymes need further investigation.

In conclusion, our study has uncovered the essential role of ATG7-dependant autophagy in maintaining the normal biological functions of human melanocytes under oxidative stress. Meanwhile, ATG7-dependant autophagy is involved in oxidative stress homeostasis by regulating ROS production, Nrf2 antioxidant signaling pathway, and the activity of several antioxidant enzymes.

To some extent, our findings have proposed a new mechanism for depigmentation skin disorders, that autophagy is indispensable for redox homeostasis and plays a crucial role in oxidative stress-induced melanocyte destruction. Further studies using appropriate depigmented animal models are required to fully clarify the role of autophagy in vivo. Besides, further identification of specific autophagy biomarkers in vitiligo patients might help find a new therapeutic target for vitiligo.

\section{Materials and methods \\ Cell culture}

Primary normal human melanocytes were isolated from human foreskin specimens obtained during circumcision surgery. The primary melanocytes were grown in Medium 254 (Cascade Biologics, Portland, OR, USA) containing Human Melanocyte Growth Supplement (Cascade Biologics). The primary melanocytes were used between the second and fourth passages in all experiments. Cells were maintained at $37{ }^{\circ} \mathrm{C}$ in a humidified atmosphere containing $5 \% \mathrm{CO}_{2}$. All subjects consented by written and informed agreement for inclusion in this study. All experiment protocols were approved by the Ethics Committee of the Department of Dermatology, Huashan Hospital, Fudan University.

\section{Lentiviral infection}

Cells were seeded at $2 \times 10^{5}$ cells per well for $24 \mathrm{~h}$ before transfection. Cells were infected with ATG7 shRNA (5'TTTGGGATTTGACACATTT-3', Genepharma, Shanghai, China) or ATG-OE (5' - AGGTCAAAGGACGAAGATAAC-3', Genepharma, Shanghai, China) at a $1: 4$ dilution in the presence of $5 \mu \mathrm{g} / \mathrm{mL}$ polybrene (Genepharma, Shanghai, China). The expression of the constructs was confirmed by qRT-PCR and western blotting.

\section{Quantitative real-time PCR}

Total cellular and tissue RNAs were isolated by the Trizol reagent (Invitrogen, Grand Island, NY) and quantified. mRNA expression was tested by using the SYBR GREEN PCR Master Mix (Applied Biosystems, Beijing, China) under a 7500 fast Real-time PCR System (Applied Biosystems). Relative expression of targeted mRNAs was calculated by the $2^{-\Delta \Delta C t}$ method, using $\beta$-actin as the internal control. All the primers were listed in Table 1.

\section{Western blotting}

Equivalent amounts of total cellular lysates $(20 \mu \mathrm{g}$ per treatment) were separated by $10 \%$ of SDS-polyacrylmide gel electrophoresis, then transferred to the polyvinylidene fluoride blots (Merck Millipore, Darmstadt, Germany). After blocking in 10\% non-fat milk, the blots were incubated with the applied primary antibodies: anti- Atg7 (Cell 
Signaling, 8558), SQSTM1/p62 (Cell Signaling, 8025), LC3A/B (Cell Signaling, 12741), GAPDH (Cell Signaling, 5174), TRP1 (Abcam, ab178676), TRP2/DCT (Abcam, ab221144), TYR (Abcam, ab170905), MITF (Abcam, ab140606), PARP (Cell Signaling, 9532), cleaved PARP (Cell Signaling, 5625), caspase 3 (Cell Signaling, 14220), cleaved caspase 3 (Cell Signaling, 9661), GCLC (Abcam, ab190685), GCLM antibody (Abcam, ab126704), NQO-1 (Cell Signaling, 62262), HO-1 (Cell Signaling, 5853), or GSTM-1/2/4/5 (Abcam, ab178684) antibodies, followed by incubation with anti-rabbit IgG, horseradish peroxidase-linked secondary antibody (Cell Signaling, 7074). Antibody-antigen binding was detected by an enhanced chemiluminescence substrate kit (Invitrogen), with the results quantified by an ImageJ software $(\mathrm{NIH}$, Bethesda, MD).

\section{Detection of apoptosis}

After experimental treatment, cells were detected by the Annexin V PE/7-AAD Apoptosis Detection Kit (BD Pharmingen, San Diego, CA) following the manufacturer's instructions and cell apoptosis was analyzed by a flow cytometry machine (Beckman Coulter, Brea, CA).

\section{SA $\beta$-galactosidase staining assay}

To measure cellular senescence, cells were stained with the SA $\beta$-Gal staining kit (Beyotime Institute of Biotechnology, Nanjing, China) according to the manufacturer's instructions. Images containing $>200$ cells were taken using a bright-field microscopy, and total and bluecolored cells were counted. The percentage of SA $\beta$-Galpositive cells was represented by the ratio between the number of blue-colored cells and the number of total cells from at least three independent experiments.

\section{Assay for reactive oxygen species levels}

Melanocytes were seeded at a density of $3 \times 10^{5} /$ well on a six-well plate at $37^{\circ} \mathrm{C}$ with $5 \% \mathrm{CO}_{2}$ for $24 \mathrm{~h}$. After $\mathrm{H}_{2} \mathrm{O}_{2}$ and UVB treatment, cells were washed twice with phosphate-buffered saline and detected by the Fluormetric Intracellular Ros Kit (BD Pharmingen, San Diego, CA). Then fluorescence was measured by flow cytometry (Beckman Coulter) within an hour. Mean fluorescence intensity was quantized with Flowjo software (Treestar USA).

\section{Statistical analysis}

Each experiment was performed at least three times and statistical analyses of the data were performed using unpaired, two-tailed Student's $t$-tests or using a two-way analysis of variance, followed by Newman-Keuls test built into GraphPad Prism (GraphPad Software 5.0; San Diego, CA). All data were expressed as mean \pm SD. $P<0.05$ were considered statistically significant.

\begin{abstract}
Acknowledgements
We acknowledge grants received from the Natural Science Foundation of China (81573064 and 81903243).
\end{abstract}

\section{Conflict of interest}

The authors declare that they have no conflict of interest.

\section{Publisher's note \\ Springer Nature remains neutral with regard to jurisdictional claims in published maps and institutional affiliations.}

Received: 18 February 2020 Revised: 7 April 2020 Accepted: 14 April 2020 Published online: 01 May 2020

\section{References}

1. Zhao, Y. et al. Autophagy is induced by UVA and promotes removal of oxidized phospholipids and protein aggregates in epidermal keratinocytes. J. Invest. Dermatol. 133, 1629-1637 (2013).

2. Zhang, C. F. et al. Suppression of autophagy dysregulates the antioxidant response and causes premature senescence of melanocytes. J. Invest. Dermatol. 135, 1348-1357 (2015).

3. Birch-Machin, M. A. \& Bowman, A. Oxidative stress and ageing. Br. J. Dermatol. 175(Suppl 2), 26-29 (2016).

4. Rinnerthaler, M., Bischof, J., Streubel, M. K., Trost, A. \& Richter, K. Oxidative stress in aging human skin. Biomolecules 5, 545-589 (2015).

5. Kruk, J. \& Duchnik, E. Oxidative stress and skin diseases: possible role of physical activity. Asian Pac. J. Cancer Prev. 15, 561-568 (2014).

6. Shah, A. A. \& Sinha, A. A. Oxidative stress and autoimmune skin disease. Eur. J. Dermatol. 23, 5-13 (2013).

7. Okayama, Y. Oxidative stress in allergic and inflammatory skin diseases. Curr. Drug Targets Inflamm. Allergy 4, 517-519 (2005).

8. Ongenae, K., Beelaert, L., van Geel, N. \& Naeyaert, J. M. Psychosocial effects of vitiligo. J. Eur. Acad. Dermatol. Venereol. 20, 1-8 (2006).

9. Laddha, N. C. et al. Vitiligo: interplay between oxidative stress and immune system. Exp. Dermatol. 22, 245-250 (2013).

10. Xie, H. et al. Vitiligo: how do oxidative stress-induced autoantigens trigger autoimmunity? J. Dermatol. Sci. 81, 3-9 (2016).

11. Guerra, L., Dellambra, E., Brescia, S. \& Raskovic, D. Vitiligo: pathogenetic hypotheses and targets for current therapies. Curr. Drug Metab. 11, 451-467 (2010).

12. Meierjohann, S. Oxidative stress in melanocyte senescence and melanoma transformation. Eur. J. Cell Biol. 93, 36-41 (2014).

13. Denat, L., Kadekaro, A. L., Marrot, L., Leachman, S. A. \& Abdel-Malek, Z. A. Melanocytes as instigators and victims of oxidative stress. J. Invest. Dermatol. 134, 1512-1518 (2014).

14. Dell'anna, M. L., Cario-Andre, M., Bellei, B., Taieb, A. \& Picardo, M. In vitro research on vitiligo: strategies, principles, methodological options and common pitfalls. Exp. Dermatol. 21, 490-496 (2012).

15. Mizushima, N. \& Komatsu, M. Autophagy: renovation of cells and tissues. Cell 147, 728-741 (2011).

16. Tobin, D. J., Swanson, N. N., Pittelkow, M. R., Peters, E. M. \& Schallreuter, K. U. Melanocytes are not absent in lesional skin of long duration vitiligo. J. Pathol. 191, 407-416 (2000).

17. Kubanov, A., Proshutinskaia, D., Volnukhin, V., Katunina, O. \& Abramova, T. Immunohistochemical analysis of 500 melanocyte content in different zones of vitiligo lesions using the Melan-A501 marker. Acta Dermatovenerol. Alp. Pannonica Adriat. 25, 5-9 (2016)

18. Ho, H. \& Ganesan, A. K. The pleiotropic roles of autophagy regulators in melanogenesis. Pigment Cell Melanoma Res 24, 595-604 (2011).

19. Ji Young, K. et al. Autophagy induction can regulate skin pigmentation by causing melanosome degradation in keratinocytes and melanocytes. Pigment Cell Melanoma Res. 33, 403-415 (2019)

20. Kumar, R., Parsad, D. \& Kanwar, A. J. Role of apoptosis and melanocytorrhagy: a comparative study of melanocyte adhesion in stable and unstable vitiligo. Br. J. Dermatol 164, 187-191 (2011).

21. Kaminskyy, V. O., Piskunova, T., Zborovskaya, I. B., Tchevkina, E. M. \& Zhivotovsky, B. Suppression of basal autophagy reduces lung cancer cell 
proliferation and enhances caspase-dependent and -independent apoptosis by stimulating ROS formation. Autophagy 8, 1032-1044 (2012).

22. Sastre, J., Pallardo, F. V. \& Vina, J. Mitochondrial oxidative stress plays a key role in aging and apoptosis. IUBMB Life 49, 427-435 (2000).

23. Eckhart, L., Tschachler, E. \& Gruber, F. Autophagic control of skin aging. Front. Cell Dev. Biol. 7, 143 (2019).

24. Duan, W. J. et al. A SIRT3/AMPKVautophagy network orchestrates the protective effects of trans-resveratrol in stressed peritoneal macrophages and RAW 264.7 macrophages. Free Radic. Biol. Med. 95, 230-242 (2016).

25. Gruber, F. et al. NF-E2-related factor 2 regulates the stress response to UVA-1oxidized phospholipids in skin cells. FASEB J. 24, 39-48 (2010).

26. Natarajan, V. T. et al. Transcriptional upregulation of Nrf2-dependent phase II detoxification genes in the involved epidermis of vitiligo vulgaris. J. Invest Dermatol. 130, 2781-2789 (2010).

27. Jian, Z. et al. Heme oxygenase-1 protects human melanocytes from $\mathrm{H}_{2} \mathrm{O} 2-$ induced oxidative stress via the Nrf2-ARE pathway. J. Invest. Dermatol. 131, 1420-1427 (2011).

28. Jiang, T. et al. p62 links autophagy and Nrf2 signaling. Free Radic. Biol. Med. 88, 199-204 (2015).

29. Ponpuak, M. et al. Delivery of cytosolic components by autophagic adaptor protein 062 endows autophagosomes with unique antimicrobial properties. Immunity 32, 329-341 (2010).
30. Jain, A. et al. p62/SQSTM1 is a target gene for transcription factor NRF2 and creates a positive feedback loop by inducing antioxidant response elementdriven gene transcription. J. Biol. Chem. 285, 22576-22591 (2010).

31. Komatsu, M. et al. The selective autophagy substrate p62 activates the stress responsive transcription factor Nrf2 through inactivation of Keap1. Nat. Cell Biol. 12, 213-223 (2010).

32. He, Y. et al. Dysregulated autophagy increased melanocyte sensitivity to H2O2-induced oxidative stress in vitiligo. Sci. Rep. 7, 42394 (2017).

33. Fischer, T. W., Kleszczynski, K., Hardkop, L. H., Kruse, N. \& Zillikens, D. Melatonin enhances antioxidative enzyme gene expression (CAT, GPx, SOD), prevents their UVR-induced depletion, and protects against the formation of DNA damage (8-hydroxy-2'-deoxyguanosine) in ex vivo human skin. J. Pineal. Res. 54, 303-312 (2013)

34. Shi, M. H. et al. Meta-analysis of the association between vitiligo and the level of superoxide dismutase or malondialdehyde. Clin. Exp. Dermatol. 42, 21-29 (2017).

35. Agrawal, S., Kumar, A., Dhali, T. K. \& Majhi, S. K. Comparison of oxidantantioxidant status in patients with vitiligo and healthy population. Kathmandu Univ. Med J. (KUMJ) 12, 132-136 (2014).

36. Xiao, B. H. et al. Glutathione peroxidase level in patients with vitiligo: a metaanalysis. Biomed. Res. Int. 2016, 3029810 (2016). 